\title{
Why Cardiac Resynchronization Therapy (CRT) is Usually Prescribed along with Automatic Implantable Defibrillation (AID)? Is it a Sensi- ble Decision? Historical Perspective
}

\author{
Caveat Emptor! \\ Max E Valentinuzzi ${ }^{1,2 *}$ \\ ${ }^{1}$ Emeritus Professor, National University of Tucumán, Argentina \\ ${ }^{2}$ Emeritus Investigator, National Research Council, Argentina
}

*Corresponding author: Max E Valentinuzzi, EE, PhD, Emeritus Professor, National University of Tucumán, Tucumán, Argentina, E-mail: maxvalentinuzzi@arnet.com.ar; maxvalentinuzzi@ieee.org

\begin{abstract}
The objective of this paper tries to answer the questions posed in the title, that is, why Cardiac Resynchronization Therapy (CRT) is usually prescribed along with Automatic Implantable Defibrillation (AID)? Is it a sensible decision? For that matter, first the origins of the rather old synchronization concept in cardiac electrophysiology are established by selectively reviewing the literature and thereafter, pinpointing when the current CRT idea came about to bring back into synchronism both ventricles of the human heart. First, it was an experimental concept discovered in lower animals that shows some resemblance with a similar phenomenon applied within the same cardiac muscle. Besides, the latter was developed slowly during the last decade of the last century shaping up in the last recent decade. The literature, however, indicates discrepancies in criteria and no clear clinical evidence to suggest a mandatory prescription of both devices. Sometimes, as other examples demonstrate, tendencies in medical practice may have dramatic and even opposite changes. Health and cardiac risk assessment and predictions have entered into a quantitative stage, but the error bands are still wide and the road ahead is quite hard. Hence, when considering the CRT-AID subject, serious reconsideration is needed.
\end{abstract}

\section{Introduction}

Normalized and highly regulated professions, like medicine and engineering, tend to follow hard rules in their respective practices, often established by certifying organisms or by highly recognized learned societies or study groups. However, if such rules are sometimes blindly applied, they may become two-edge swords, be- ing beneficial with one and harmful with the other. The above aphorism, from Latin caveat (or may he beware), is a subjunctive form of cavere (to beware) and emptor (buyer); thus, it simply states let the buyer beware. Lawyers are familiar with this rather old statement.

The caveat notifies a buyer that the goods he or she is buying are possibly subject to defects. The purchaser assumes the risk that the product might be either defective or unsuitable to his or her needs. Buyers typically have less information about the good or service they are purchasing, while the seller has more information. The quality of this situation is known as information asymmetry. Defects in the good or service may not be known by the buyer and only known to the seller.

A medical service, although not the same as a commercial transaction, is somewhat analogous to a seller-buyer act. However, its information asymmetry is enormous and even worse, for it can be asked if always the physician is fully aware of what he/she is offering, especially when the rule is blindly applied. The case we deal with here refers to whether CRT really requires a simultaneous AID, lest it may be deemed a malpractice, a serious accusation no physician would want to run into. This article questions such stand, favoring CRT without AID, unless it were absolutely demonstrated and even more if the patient for some reason request the latter (that is, only CRT). Hence, the objective herein tries to answer the questions posed in the title, selectively re- 
viewing the literature and bringing up other relevant aspects, too.

\section{Cardiac Synchronization}

In the normal cardiac situation both ventricles show a minor physiological dyssynchrony, i.e., the right ventricular contraction starts a little after that of the left, and such delay lies in the order of $15-25 \mathrm{~ms}$, at most even 30 in few cases [1]. In some cardiopathies, this delay may increase substantially. There are three types of dyssynchronies, say,

A) Atrioventricular (AV), showing long delays;

B) Interventricular or RV to LV contraction and;

C) Intraventricular or segmental disruption of LV wall activation and contraction.

The first Type A occurs when the delay appears between atrial and ventricular contraction. It produces shortened ventricular filling time as well as superposition of atrial contraction on early passive filling, both of which reduce LV filling.

The second Type B occurs when there is a delay between RV and LV activation. It is likely in the presence of Left Bundle Branch Block (LBBB), in which RV contraction precedes LV contraction (opposite of the healthy case), leading to abnormal septal motion, uncoordinated LV contraction and a decreased LV ejection fraction. One criterion of assessing it is the QRS width, which shows a normal range of $80-120 \mathrm{~ms}$. When that duration surpasses $140 \mathrm{~ms}$, the two ventricles are considered as contracting out of physiological synchrony and call for correction or resynchronization.

The third Type $C$ takes place when there is uncoordinated contraction of several LV segments. It results in LV wall segments contracting early, not contributing to ejection of blood and late segments producing higher wall stress.

No doubt, all three types have adverse effects on the mechanical efficiency of the heart. A didactic gross comparison reminds of a gasoline engine working out of tune, which in simple words means its sparkplugs in the cylinders do not rightly ignite following the normal sequence for an efficient running. The correction procedure is called tune up. Hence and keeping prudent distance, it may be said that the CRT "tunes up" the ventricles [2-4]. Cardiac resynchronization tends to restore contractile coordination due to conduction delays. For that matter, the ventricular region with the most delayed mechanical activation is stimulated to contract in synchrony with the earlier stimulated area. Two leads at least are thus employed, one to each ventricle.

\section{Overall Concept of Synchronization: Background}

The concept of synchronism and synchronization in cardiac physiology goes at least as far back as 1946, when an experimental paper by Marcel Segers, from the Insti- tute Solvay of Physiology, in Brussels, Belgium, introduced the concept of cardiac synchronization of two frog's hearts when placed in close vicinity [5]. The fastest slightly decreased its rate while the slower one increased it. Perhaps the words entrainment or coupling better describe the phenomenon. His translated own words should be quoted selecting some paragraphs from that paper.

"One of the very typical consequences of vicinity influences resides in the interneuronal synchronization phenomenon put into evidence by Adrian (1930). In the present work, we wish to establish whether such synchronization can develop in the frog's heart; hence, we have studied the possible interactions between two different centers of automatism when they are not linked by any anatomical structure. We put in contact two different frog cardiac fragments (Rana esculenta and Rana temporaria) cut either at the level of the auricles or at the level of the ventricles. The two pieces were placed side by side or one over the other, on the surface of a cotton pad soaked with Ringer solution while their electric activity was recorded by a polygraphic technique. Synchronization takes place rather frequently when the difference between the two rhythms is smaller than $5 \%$, essentially, the slower fragment accelerates while the faster one slows down".

The final summary given by Segers says in its three first and most significant points:

1. Two juxtaposed cardiac pieces can synchronized when their beats show close frequencies; synchronization sets by and large on an intermediate frequency.

2. Both synchronized pacemakers continue their respective activations over their particular territories but keeping only a very weak of independence one from the other.

3. The relationship between both rhythms can show different values: $1 / 1,2 / 1,3 / 1,4 / 1,3 / 2$. Sometimes, synchronization persists just a few contractions. It then happens an accrochage (coupling) of the two rhythms. A preliminary note was published in Acta Biologica Belgica, in 1943, of the Biological Society of Belgium, Bruxelles.

In another paper published a year later, Segers and coworkers reported that during complete heart block the auricular and ventricular rhythms may in some cases remain synchronized in a 2:1 ratio during very long periods. After exercise, the synchronization disappears, but a close association of a P-wave and an R-wave (phénomène $d^{\prime}$ accrochage or coupling phenomenon) may occur. These facts must be attributed to neighborhood interreactions developing between ventricles and auricles, without any conduction pathway. They are similar to the synchronization which is observed when two frog hearts are placed in contact, as reported previously by the same Segers. It was concluded that the rhythms of auricles and ventricles are not necessarily independent during complete heart block [6]. 
A similar observation was described years later by Marriott [7]: Complete heart block implies an independence between atria and ventricles that does not always exist. Segers, as referred to above, showed that, after complete block was artificially produced in the frog's heart, atria and ventricles would sometimes begin to beat exactly in phase, most commonly in a 2 to 1 ratio. He subsequently reported one clinical example of 2 to $1 \mathrm{~A}-\mathrm{V}$ synchronization in a patient with complete heart block. Two further cases that may illustrate different varieties of synchronization were presented in this latter 1956 paper.

The conclusion of the preceding paragraphs clearly says that the concept of cardiac synchronization, loosely stated, has a history of over 70 years. Is it related to the current concept of CRT? When did CRT start? Who, where? It is difficult to pin point exactly the answers to these questions for the resynchronization idea apparently was in the cardiological air, although not clearly set, even considering the background material given above showing some similarities. None the less and as far back as we could ascertained, perhaps the ventricular resynchronization concept began to shape up during the 1990-2001 period and that is, considering one ventricle with respect to the other, entities with obvious anatomical links.

There is a description of the beneficial effects of physiologic dual-chamber pacing in the treatment of end-stage idiopathic dilated cardiomyopathy in 16 patients in whom conventional drug therapy had failed. Candidates for cardiac transplantation as well as patients not accepted for transplantation participated. During pacing at an atrioventricular delay of $100 \mathrm{~ms}$, left ventricular ejection fraction increased from $16.0 \pm$ 8.4 to $25.6 \pm 8.6 \%$ accompanied by a striking improvement in clinical symptoms, such as severe dyspnea at rest and pulmonary edema. There was a decrease in preload, too. Normalization of heart rate was achieved. All patients returned to a relatively normal life. Within 1 year after onset of pacing only 4 of the patients died. The conclusion was that pacing could represent an alternative approach for chronic heart failure due to dilated cardiomyopathy who no longer respond to drug therapy [8]. Another significant reference is the DAVID Trial [9]. Several other papers appeared about dual-chamber pacing applied to congestive heart failure during the last decade of the former century; in fact and without expressly saying it, all aimed at correcting rightleft ventricular dyssynchrony, but mostly centering the criterion on the A-V delay [10-19]. Observe that the last reference comes from a multicenter clinical study, and it is over 17-years-old. It seems to have been the first organized clinical trial about the subject.

Cazeau, Leclercq, et al. [20] in turn, in 2001 and following a line of thought similar to the previous researchers, found that one third of patients with chronic heart failure have electrocardiographic evidence of a major in- traventricular conduction delay, which may worsen left ventricular systolic dysfunction through asynchronous ventricular contraction. Apparently, they put forward such concept for the first time. Uncontrolled studies suggested that multisite biventricular pacing improved hemodynamics by reducing ventricular asynchrony. Thus, sixty-seven patients with severe heart failure due to chronic left ventricular systolic dysfunction, with normal sinus rhythm and a duration of the QRS interval of more than $150 \mathrm{msec}$, received transvenous atrio-biventricular pacemakers. They concluded that atrio-biventricular pacing significantly improved exercise tolerance and quality of life in patients with chronic heart failure and intraventricular conduction delay [20].

Already within this new century, the number of contributions increased greatly and at a growing rate until establishing Cardiac Resynchronization Therapy (CRT) as an accepted procedure. At one moment during the last 15 years or so, it was decided to recommend CRT with an AID. Hence, another question arises: How high the probability of ventricular fibrillation/tachyarrhythmia risk is in the presence of a long QRS? What the experience has been so far? These doubts do not have yet a firm response.

\section{Ventricular Fibrillation Risks}

The evaluation of health risk has been a question of high concern both for patients and physicians since ancient times, eventually wanting to know when death would take us away. In the case of the heart, for which several more or less recent indices are available $[21,22]$ most of the events end up in ventricular fibrillation [23]. The human heart is inhomogeneous in its anatomical and histological structure and such fact facilitates its falling into the arrhythmia, easily lethal if not attended as a right-now-emergency. Homogeneous ventricles, instead, like the herptilian heart, rarely if ever show fibrillation, which usually is described as rapid asynchronic or uncoordinated contractions $[24,25]$. The latter two references give evidence of these peculiarities and also possibly relate the phenomenon to non-linear chaotic behavior. It must be underlined that an added or extra inhomogeneity to the already mammalian inhomogeneous tissue, such as ischemia, or an electric shock, or a mechanical disturbance, may trigger the arrhythmia [26].

\section{Discussion}

Let us first review some papers published in the last ten years or so to proceed, thereafter, with other aspects that usually are not being considered, probably because the subject has become enclosed within a shielded procedural-protocolar case that no one dares invade.

In 2004, Bristow, Saxon, et al. [27] in a group of selected patients, studied cardiac-resynchronization therapy alone and in conjunction with an automatic implant- 
ed defibrillator. They stated that the decision of which of these two therapeutic options is appropriate for a particular setting is best determined on an individual basis by patients and their physicians [27]. The final sentence prudently leaves the decision to the patient-physician, that is, not necessarily CRT must be accompanied by an AID. Even though these investigators explain that "eligible patients who provided written informed consent were randomly assigned to treatment with protocol-mandated optimal pharmacologic therapy alone, optimal pharmacologic therapy plus cardiac-resynchronization therapy with a pacemaker or optimal pharmacologic therapy plus cardiac-resynchronization therapy with a pacemaker-defibrillator", the interpretation given by the present article is of doubt, that is, the authors seem to have had covert doubts. Too many conditions tend to add confusion.

Kadish and Mehra, in 2005 [28] said: "It is unclear whether an ICD must accompany a cardiac resynchronization device or whether it should be performed in isolation. Taking recent results in context, one could argue that CRT therapy should ideally be accompanied by ICD placement in those with NYHA III symptoms. Results also suggest that ICDs might be beneficial in heart failure patients with advanced symptoms, irrespective of ischemic or nonischemic etiology [28]. Again doubts ... unclear, ideally ...

In 2007, a very comprehensive report was produced in Italy [29], however, no reference was made regarding the subject dealt with in this paper. The authors repeat what we just mentioned before, i.e., that ventricular mechanical dyssynchrony plays an important role in pathological conditions such as hypertrophy, ischemia, infarction or heart failure, adding that several prospective randomized controlled trials supported the clinical efficacy and safety of Cardiac Resynchronization Therapy (CRT) in patients with moderate or severe heart failure and ventricular dyssynchrony. Nothing new, in fact, although a very informative piece bringing the conductance catheter as another tool to evaluate the level of dyssynchrony.

In turn, Ganjehei, Razavi and collaborators [30] report that despite the major improvements achieved by CRT, only about one third of HF patients qualify for CRT. Furthermore, one third of patients who receive CRT do not respond favorably to it and their condition might indeed worsen. Criteria for CRT: Optimal medical therapy in the presence of QRS duration > 120 ms, LV Ejection Fraction (LVEF) $\leq 0.35$ and New York Heart Association (NYHA) functional class III or stable functional class IV. Improvement in the 6-minute walk, oxygen consumption and frequency of hospitalizations for HF were found in all studies. Echocardiographic measures of ventricular dyssynchrony often are unable to distinguish responders from non responders. Despite remarkable advances with CRT over the last decade, the problem of identi- fying those patients who will not respond favorably to CRT (non responders) remains. Cardiac resynchronization therapy improves quality of life, increases exercise tolerance and reduces hospitalizations for patients with HF. Furthermore, CRT reverses remodeling of the LV by decreasing LV volume and increasing LVEF. Cardiac resynchronization therapy is beneficial even in mildly symptomatic or asymptomatic patients who otherwise meet the criteria for CRT [30]. Nothing is said about AID in this paper.

Ouellet, Huang and collaborators, in 2012 [31] after a study, concluded that although CRT-D does reduce the risk of a first tachyarrhythmia event in LBBB patients, it does not reduce the risk of subsequent recurrent events. In addition, CRT-D may increase the risk of subsequent tachyarrhythmias in patients without LBBB [31]. Again, nothing certain regarding the questions posed for this article.

The paper by Kutyifa, Pouleur and collaborators [32] is more direct in assessing the subject as they aimed at evaluating the relationship between Left Ventricular (LV) dyssynchrony and the risk of Ventricular Tachycardia (VT) or Ventricular Fibrillation (VF). They underlined that intraventricular mechanical dyssynchrony might be an important factor in ventricular arrhythmogenesis by enhancing electrical heterogeneity in heart failure patients. Besides, it is pointed out that the effects of dyssynchrony have not yet been evaluated in a large cohort of ICD and Cardiac Resynchronization Therapy with Defibrillator (CRT-D) patients. The paper states: Patients without $\angle B B B$ receiving CRT-D did not show reduction in VT/VF/death or in VT/VF in relation to improving dyssynchrony when evaluating cumulative event rates or risk of events. In conclusion: Baseline LV dyssynchrony did not predict VT/VF/death or VT/VF in mild heart failure patients with or without LBBB. CRT-induced improvement of LV dyssynchrony was associated with significant reduction of ventricular arrhythmias in patients with LBBB. In other words, doubts continue, even with some not too impressive improvements [32]. No clear contribution to our question either in this paper.

A more recent paper says that data on the time-dependent benefit of Cardiac Resynchronization Therapy with Defibrillator (CRT-D) compared with a dual-chamber Implantable Cardioverter-Defibrillator (ICD) to reduce death or Ventricular Tachycardia (VT) or Ventricular Fibrillation (VF) are limited. They aimed at evaluating the time-related risk of death or sustained VT or VF in patients receiving CRT-D vs. ICD in the MADIT-RIT trial. Of the ICD $(n=742)$ and CRT-D ( $n=757)$ patients enrolled, the risk of death was lower in CRT-D vs. in ICD early after device implantation and beyond 6 months of follow-up. The overall risk of sustained VT/VF was reduced in CRT-D vs. ICD patients. However, the risk was similar in the first 6 months and a lower risk emerged 6 months after CRT-D implantation. Patients on guideline-based treatment with a Cardioverter-Defibril- 
lator combined with Resynchronization Therapy (CRT-D) have a lower risk to die or to experience a first episode of Ventricular Tachycardia (VT)/Ventricular Fibrillation (VF) compared with patients on guideline-based treatment with an Implantable Cardioverter-Defibrillator (ICD) alone. The favorable effect of guideline-based CRT-D vs. a guideline-based ICD alone on mortality starts early and is continued during follow-up. Guideline-based treatment with CRT-D vs. a guideline-based defibrillator alone is associated with lower risk of VT/VF. Guideline-Based Treatment is a standardized protocol, which are used in many medical domains. This favorable effect of CRT-D is not detectable until 6 months of treatment. However, nothing is said in this paper about CRT alone and the paper does not give a definitive answer to our question. Thus, doubts continue [33].

Since remodeling is mentioned in some of the above mentioned papers, this is a good point to clarify the concept which usually appears confusing. Cardiac remodeling refers to the changes in size, shape, structure and physiology of the heart after injury to the myocardium. The injury is typically due to acute myocardial infarction, but may be from a number of causes that result in increased pressure or volume overload (wall strain increases). There are other causes, too. A series of histopathological and structural changes occur in the left ventricular myocardium that lead to progressive decline in its performance. Ultimately, remodeling may result in diminished contractile (systolic) function and reduced stroke volume. Medically speaking, ventricular remodeling implies a decline in function (even though the word remodeling usually implies improvement, obviously confusing). The term reverse remodeling implies an improvement in ventricular mechanics and function following a remote injury. Then, we find situations like concentric hypertrophy, due to pressure overload and eccentric hypertrophy, which recognizes volume overload as its cause. Heart Failure (HF) involves changes in cardiac structure, myocardial composition, myocyte deformation and multiple biochemical and molecular alterations that impact cardiac function and reserve capacity. Collectively, these changes have been referred to as cardiac remodeling. Reverse remodeling appears as its opposite concept and is successfully achieved by inhibitors of the renin-angiotensin-aldosterone system, $\beta$-blockers and device therapies such as cardiac resynchronization or ventricular assist devices. Reverse remodeling is defined by lower chamber volumes (particularly end-systolic volume) and is often accompanied by improved $\beta$-adrenergic and heart-rate responsiveness. At the cellular level, reverse remodeling impacts on myocyte size, function, excitation-contraction coupling, bioenergetics and a host of molecular pathways that regulate contraction, cell survival, mitochondrial function, oxidative stress and other features [34]. How much the remodeling or reversal remodeling processes influence the risk of tachyarrhythmias? There are no answers to this question.
There are aspects that need to be taken into account for a better evaluation of this elusive and rather controversial subject. One of them recalls the concept of the QRS complex as the difference resultant of two ventricular monophasic action potentials, one shifted with respect to the other [35]. The shift between such potentials precisely defines the QRS duration (width), the longer the shift, the longer the QRS, so providing a good explanation of the phenomenon to be looked at.

Another question: Is ventricular dyssynchrony an inhomogeneity? At first sight, it would qualify as such and, therefore, it would favor triggering of irregular fast arrhythmias, which in turn would justify an associated AID to the CRT. None the less, at second thoughts, this inhomogeneity seems to be of a different kind as compared to those produced, say, by ischemia or blocks, or by electric or mechanical disturbances. The information collected from clinical trials is very inconsistent and incomplete in this respect.

A patient with an implanted defibrillator, knowing of the possibility of a discharge at any circumstance may live under a state of constant fear, even more if he/she went into the experience. The design of AID's includes a detection algorithm, the sooner the detection of the arrhythmia, the sooner the defibrillating shock and the better the probability of success but, if detection is too soon, the shock may be delivered when the patient is still conscious and the pain produced is unbearable. It has been described as a tremendous kick in the back. There are documented experiences, not publicized, when thereafter the patient firmly requested removal of the device in spite of the risk of possible death. They reported not being able to sleep at night because they seemed to feel the discharge. The author of this article received when a young student a high voltage shock that produced respiratory arrest but not fibrillation; during months after, he many times felt over and over again at night the pain. Not nice! Besides, such an early detection may not be a reliable one for it is based in a very short period, perhaps 3-6 s, at most. Was that reading time enough to label the signal as fibrillation or tachycardia? Clearly not. In other words, the AID may detect a false positive delivering an unnecessary discharge and even running the risk of triggering the arrhythmia.

When one inspects in retrospect certain specific trends in clinical and surgical procedures, changes are easily detected, sometimes influenced by wrong ideas or lack of scientific knowledge or perhaps by established protocols originated in older practices or customs or beliefs. We will briefly mentioned three examples, but others may be found if digging deeper.

\section{Cesarean operations}

In 2007, nearly one-third (32\%) of all births were cesarean deliveries. Although there are often clear clinical indications for a cesarean delivery, the short- and longterm benefits and risks for both mother and infant have 
been the subject of intense debate for over 25 years. Cesarean delivery involves major abdominal surgery and is associated with higher rates of surgical complications and maternal rehospitalization, as well as with complications requiring neonatal intensive care unit admission. In addition to health and safety risks for mothers and newborns, hospital charges for a cesarean delivery are almost double those for a vaginal delivery, imposing significant costs. This report shows trends in cesarean delivery since 1991, focusing on the period from 1996 to 2007 when cesarean rates began to rise following a decline in the early 1990s [36]. Apparently, the word derives from the surgical birth of Julius Caesar, directly from the abdomen of his mother Coronis, but nothing of this is certain.

\section{Bloodletting}

It was the withdrawal of blood from a patient to cure or prevent illness and disease. It was based on an ancient concept in which blood and other bodily fluids were regarded as humors that had to remain in proper balance. It probably was the most common practice performed by surgeons from antiquity until the late $19^{\text {th }}$ century or even first quarter of the 1900's. The practice has now been abandoned by modern medicine.

\section{Polio vaccines}

The saga behind their development is outstanding and heart breaking and too long. In a way, both ever confronting scientists, Albert Sabin (1906-1993) and Jonas Salk (1914-1995), ended up by having been right, although they never knew it. Both died of heart failure. The CDC Advisory Committee recommended in 1996 a mixed program using both vaccines (attenuated, proposed by Sabin and dead virus, advocated by Salk), including some special remarks to be taken into account [37].

The point to underline from the above mentioned examples refers to how in medicine, ideas, concepts, trends, change over time, as knowledge proceeds, often paying a high toll. The final aspect to consider is the patient's view, as best informed as possible, in cases without understanding exactly what his/her own health situation is, in other cases with full knowledge, but in any event highly respectful simply because it refers to his/ her body. Reference [26] must be recalled for it offers an excellent and similar position in this regard. Finally and in addition, CRT + Pacing (CRT-P) is common outside the USA, whereas CRT + ICD (CRT-D) is most common in the USA, pointing out perhaps to subjective differences in criteria rather than plain objectivity.

\section{Conclusions}

There is no doubt that health and cardiac risk assessment and predictions have entered into a quantitative stage, but the error bands are still wide and the road ahead is quite hard. Are we not falling perhaps into a comparable situation recalling the three examples given above, when considering the CRT-AID subject here in discussed? This article suggests a serious revision, even recognizing several technological improvements that have added safety and reliability.

\section{References}

1. Hurst JW (2003) A new terminology for describing left and right ventricular conduction abnormalities. Clin Cardiol 26: 540-545.

2. Bax JJ, Ansalone G, Breithardt OA, Derumeaux G, Leclercq C, et al. (2004) Echocardiographic evaluation of cardiac resynchronization therapy: Ready for routine clinical use? A critical appraisal. J Am Coll Cardiol 44: 1-9.

3. Kass DA (2003) Predicting cardiac resynchronization response by QRS duration: The long and short of it. Journal of the American College of Cardiology 42: 2125-2127.

4. Mirvis D, Goldberger AL (2015) Electrocardiography. In: Mann DL, Zipes DP, Libby P, WB Saunders Co, Braunwald's Heart Disease: A Textbook of Cardiovascular Medicine. (10 $10^{\text {th }}$ edn), Philadelphia, USA.

5. Segers M (1946) Les phenomenes de synchronisation au niveau du coeur. Archives Internationales de Physiologie 54: $87-106$

6. Segers M, Lequime J, Denolin H (1947) Synchronisation of auricular and ventricular beats during complete heart block. American Heart J 33: 685-691.

7. Marriott HJL (1956) Atrioventricular synchronization and accrochage. Circulation 14: 38-43.

8. Hochleitner M, Hörtnagl H, Ng CK, Hörtnagl H, Geschnitzer $F$, et al. (1990) Usefulness of physiologic dual-chamber pacing in drug-resistant idiopathic dilated cardiomyopathy. Am J Cardiol 66: 198-202.

9. The DAVID Trial Investigators (2002) Dual-Chamber pacing or ventricular back-up in patients with implantable defibrillator. The Dual chamber and VVI Implantable Defibrillator (DAVID) trial. JAMA 288: 3115-3123.

10. Brecker SJD, Xiao HB, Sparrow J, Gibson DG (1992) Effects of dual-chamber pacing with short atrioventricular delay in dilated cardiomyopathy. Lancet 340: 1308-1312.

11. Hochleitner $M$, Hörtnagl $H$, Hörtnagl $H$, Fridrich L, Gschnitzer F (1992) Long-term efficacy of physiologic dual-chamber pacing in the treatment of end-stage idiopathic dilated cardiomyopathy. Am J Cardiol 70: 1320-1325.

12. Bakker PF, Meijburg $H$, de Jonge $N$, van Mechelen $R$, Wittkampf $F$, et al. (1994) Beneficial effects of biventricular pacing in congestive heart failure. Pacing Clin Electrophysiol 17: 820.

13. Gold MR, Feliciano Z, Gottlieb SS, Fisher ML (1995) Dual-chamber pacing with a short atrioventricular delay in congestive heart failure: A randomized study. J Am Coll Cardiol 26: 967-973.

14. Linde C, Gadler F, Edner M, Nordlander R, Rosenqvist M, et al. (1995) Results of atrioventricular synchronous pacing with optimized delay in patients with severe congestive heart failure. Am J Cardiol 75: 919-923.

15. Nishimura RA, Hayes DL, Holmes DR, Tajik AJ (1995) Mechanism of hemodynamic improvement by dual-chamber pacing for severe left ventricular dysfunction: an acute doppler and catheterization hemodynamic study. J Am Coll Cardiol 25: 281-288.

16. Auricchio A, Salo RW (1997) Acute hemodynamic improvements by pacing in patients with severe congestive heart 
failure. Pacing Clin Electrophysiol 20: 313-324.

17. Shinbane JS, Chu E, DeMarco T, Sobol Y, Fitzpatrick AP, et al. (1997) Evaluation of acute dual chamber pacing with a range of atrioventricular delays on cardiac performance in refractory heart failure. Journal of the American College of Cardiology 30: 1295-1300.

18. Saxon LA, Kerwin WF, Cahalan MK, Kalman JM, Olgin JE, et al. (1998) Acute effects of intraoperative multisite ventricular pacing on left ventricular function and activation/ contraction sequence in patients with depressed ventricular function. J Cardiovasc Electrophysiol 9: 13-21.

19. Auricchio A, Stellbrink C, Block M, Bakker P, Sack S, et al. (1999) The Pacing Therapies for Congestive Heart Failure (PATH-CHF) Study: Rationale, design and endpoints of a prospective randomized multicenter study. Am J Cardiol 83: $130-135$

20. Cazeau S, Leclercq C, Lavergne T, Walker S, Varma C, et al. (2001) Effects of multisite biventricular pacing in patients with heart failure and intraventricular conduction delay. $\mathrm{N}$ Engl J Med 344: 873-880.

21. Gilbert K, Larocque BJ, Patrick LT (2000) Prospective evaluation of cardiac risk indices for patients undergoing noncardiac surgery. Ann Intern Med 133: 356-359 (TIMI).

22. Thrombolysis In Myocardial Ischemia or Infarction.

23. Valentinuzzi ME, Arini PD, Laciar E, Bonomini MP, Correa RO (2013) Cardiac risk assessment: When and Who? IEEE Pulse 4: 38-48.

24. Savino GV, Valentinuzzi ME (1988) Ventricular fibrillation-defibrillation in the toad Bufo paracnemis. Int J Cardiol 19: 19-25.

25. Savino GV, Romanelli L, González DL, Piro O, Valentinuzzi ME (1989) Evidence for chaotic behavior in driven ventricles. Biophys J 56: 273-280.

26. Valentinuzzi ME (2010) Fibrillation-Defibrillation: Clinical and Engineering Aspects. 8 chapters, Series on Bioengineering \& Biomedical Engineering, World Scientific Publisher (WSP), New Jersey \& Singapore.
27. Bristow MR, Saxon LA, Boehmer J, Krueger S, Kass DA, et al. (2004) Cardiac-Resynchronization Therapy with or without an Implantable Defibrillator in advanced chronic heart failure. N Engl J Med 350: 2140-2150.

28. Kadish A, Mehra M (2005) Heart failure devices: Implantable Cardioverter-Defibrillators and Biventricular Pacing Therapy. Circulation 111: 3327-3335.

29. Valsecchi S, Censi F, Bartolini P (2007) Methods for the Evaluation of the Ventricular Mechanical Dyssynchrony: State of the Art. Istituto Superiore di Sanità, Italia.

30. Ganjehei L, Razavi M, Massumi A (2011) Cardiac Resynchronization Therapy: A decade of experience and the dilemma of nonresponders. Tex Heart Inst J 38: 358-360.

31. Ouellet G, Huang DT, Moss AJ, Hall WJ, Barsheshet A, et al. (2012) Effect of Cardiac Resynchronization Therapy on the risk of first and recurrent ventricular tachyarrhythmic events in MADIT-CRT. J Am Coll Cardiol 60: 1809-1816.

32. Kutyifa V, Pouleur AC, Knappe D, Al-Ahmad A, Gibinski $M$, et al. (2013) Dyssynchrony and the risk of ventricular arrhythmias. JACC Cardiovascular Imaging 6: 432-444.

33. Stockburger M, Moss AJ, Olshansky B, Klein H, McNitt S, et al. (2015) Time-dependent risk reduction of ventricular tachyarrhythmias in cardiac resynchronization therapy patients: a MADIT-RIT sub-study. Europace 17: 1085-1091.

34. Koitabashi N, Kass DA (2011) Reverse remodeling in heart failure: Mechanisms and therapeutic opportunities. Nat Rev Cardiol 9: 147-157.

35. Geddes LA, Baker LE (1989) Principles of Applied Biomedical Instrumentation. ( $3^{\text {rd }}$ edn), John Wiley \& Sons, New York, USA, 992.

36. Menacker F, Hamilton BE (2010) Recent Trends in Cesarean Delivery in the United States. NCHS Data Brief 1-8.

37. Oshinsky DM (2005) POLIO: An American Story. Oxford University Press, New York, 342. 